\title{
Preparation, Characterization and Theoretical Study of a New Chromate Ionic liquid: Triphenylphosphonium Tetrafluorochromate
}

\author{
S. Ghammamy ${ }^{1}$, K. Mehrani ${ }^{2}$, G. Geimechi ${ }^{2}$, and S. Sedaghat ${ }^{1}$ \\ ${ }^{1}$ Department of Chemistry, Islamic Azad University, Malard Branch, Malard, Iran \\ ${ }^{2}$ Department of Chemistry, Islamic Azad University, Ardabil Branch, Ardabil, Iran
}

Received 2 February 2012, accepted in final revised form 25 June 2012

\begin{abstract}
The synthesis of triphenylphosphonium tetrafluorochromate (III) liquid (IL) is reported here. The product was characterized by spectroscopic and analytical methods such as, FTIR, cyclic voltammetry, CHN, TGA and differential thermal analysis (DTA). Thermal decomposition of this compound showed multistage processes and also results show that this IL has excellent thermal stability below $100^{\circ} \mathrm{C}$. In this paper, the optimized geometries and frequencies of the stationary point and the minimum-energy paths were calculated by using the DFT (B3LYP) methods with $6-311 \mathrm{G}^{*}$ basis sets. From these calculations, optimized geometries, molecular parameters, and vibrational spectra of IL have been calculated. In addition, calculated frequencies were compared with the experimental frequencies after correction by the appropriate scaling factor. This comparison showed that our theoretical data are in good agreement with the experimental results.
\end{abstract}

Keywords: Chromate; DFT; Frequency; Ionic liquid; Room temperature.

(C) 2012 JSR Publications. ISSN: 2070-0237 (Print); 2070-0245 (Online). All rights reserved.

doi: http://dx.doi.org/10.3329/jsr.v4i3.9915 J. Sci. Res. 4 (3), 649-656 (2012)

\section{Introduction}

There is an inverse proportionality between industrial importance and scientific interest in the case of chromium compounds. It is a simple salt which dissociates in solution and whose properties have, with a few exceptions, been known for many years. Room temperature ionic liquids have generated considerable excitement in recent years as a new type of solvent media that possesses minimal vapor pressure. At the end of twentieth century, scientists found that unlike traditional view about the melting points of salts, there is a class of salts or salt mixtures those have melting points below $100^{\circ} \mathrm{C}_{2}$ which are referred as ionic liquids [1]. Room-temperature ionic liquids (RTIL) [2] are ionic liquids

\footnotetext{
*Corresponding author: shghamami2012@yahoo.com
} 
with melting points at or below room temperature [3]. Some ionic liquids (RTIL) are nonflammable, nonvolatile, or thermally stable and can be used as a promising replacement for the traditional organic solvents. Moreover, many workers have synthesized and studied about ionic liquids. The ionic liquids in organic reaction are often obtained as organic product and can be removed easily from the ionic liquid by extraction with organic solvent without resorting to an aqueous workup or solvent evaporation.

It is noteworthy that ionic liquids have been applied for many other purposes. For example, during past years, ionic liquids have been applied to synthesize different compounds [4], polymerization processes, dye synthesis, supercritical fluid chromatography, determination of phenothiazine derivatives, preparation of sensors and biosensors, preparation of nanostructures, and other applications [5].

However, recent reports indicated that several ionic liquids have been applied to separate various mixtures [6]. Moreover, ionic liquid properties such as heat capacities and refractive index [7], luminescence properties [8], osmotic coefficients [9], enthalpy, density, heat capacity [10], and thermo physical properties [11] have been studied since their first synthesis. Therefore, following our previous studies on chemistry of ionic liquids', we decided to improve our knowledge about these compounds by synthesis, characterization, and theoretical study of a new borate-based ionic liquid. This is useful when a metal catalyst is used in the reaction that is this catalyst often remains in the ionic liquid and can be directly reused. In addition, ionic liquids as a class of novel environmental "green solvents," have remarkable new properties and promising applications in many fields. The first RTIL, ethyl ammonium nitrate (M.P.: 13-14 ${ }^{\circ}$ ), was reported in 1914. However, ionic liquids did not draw much attention till 1992, when Wilkes and coworkers reported air- and water-stable RTILs based on imidazolium salts. Subsequently, research on their synthesis, properties, and applications has increased substantially.

In this work, the synthesis, structural, bonding, thermal solubility, and vibrational properties, and computational study of new chromium ionic liquid has been reported. From the results, calculated molecular parameters and vibrational frequencies are shown.

\section{Experimental}

\subsection{Materials and Instruments}

Starting materials were obtained from Merck and were used without further purification. Solvents were purified by standard methods. Organic solvents were reagent grade. Voltammetry was obtained in two different velocities. The IR spectrum was recorded using FTIR Bruker Tensor 27 spectrometer. All the chemical shifts are quoted in ppm using the high-frequency positive convention and reference to TMS. The percent composition of elements was obtained from the Micro analytical Laboratories. 


\subsection{Synthesis of triphenylphosphonium tetrafluorochromate $\left[\mathrm{P}\left(\mathrm{C}_{6} \mathrm{H}_{5}\right)_{3} \mathrm{H}\right]^{+}[\mathrm{CrF}]^{-}$}

Triphenylphosphonium tetrafluorochromate (III) $\left[\mathrm{P}\left(\mathrm{C}_{6} \mathrm{H}_{5}\right)_{3} \mathrm{H}\right]^{+}\left[\mathrm{CrF}_{4}\right]^{-}$was prepared as follows: In a $100-\mathrm{mL}$ round-bottom flask, dry triphenylphosphine $(0.32 \mathrm{~g}, 1.22 \mathrm{mmol})$ was dissolved in dry acetonitrile $(20 \mathrm{~mL})$ and was stirred for $30 \mathrm{~min}$. While the mixture was stirred, (0.02 g, $1.00 \mathrm{mmol}) \mathrm{HF}$ was added and stirring continued for $5 \mathrm{~min} . \mathrm{CrF}_{3}$ $(0.14 \mathrm{~g}, 1.16 \mathrm{mmol})$ in acetonitrile was added to this mixture as the last of starting materials and stirring continued for $4 \mathrm{~h}$ to precipitate a white solid. A light green precipitate was filtered and washed with ether and hexane. M.P.: $105-106^{\circ} \mathrm{C}$; Anal. Calc. for $\left[\mathrm{P}\left(\mathrm{C}_{6} \mathrm{H}_{5}\right)_{3} \mathrm{H}\right]^{+}\left[\mathrm{CrF}_{4}\right]^{-}$: Calculated C, 48.21; H, 3.57. Found: C, 48.39; H, 3.77. IR ( $\left.\mathrm{KBr}\right)$ $\left(\mathrm{cm}^{-1}\right)$ : 2240, 851, 1471, 1579, 3057, 1426, 497, 542, 607, $687 \mathrm{~cm}^{-1}$ (Fig. 1, Table 1).

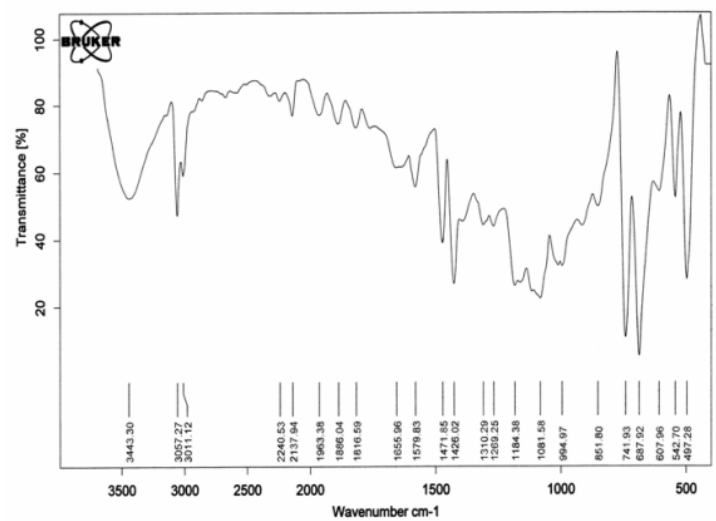

Fig. 1. FTIR spectrum of $\left[\mathrm{P}\left(\mathrm{C}_{6} \mathrm{H}_{5}\right)_{3} \mathrm{H}\right]^{+}\left[\mathrm{CrF}_{4}\right]^{-}$.

Table 1. Experimental frequencies of $\left[\mathrm{P}\left(\mathrm{C}_{6} \mathrm{H}_{5}\right)_{3} \mathrm{H}\right]^{+}\left[\mathrm{CrF}_{4}\right]^{-}\left(\mathrm{cm}^{-1}\right)$.

\begin{tabular}{rll}
\hline$v\left(\mathrm{~cm}^{-1}\right)$ & Vibration & Intensity \\
\hline & P( $\left.\mathrm{C}_{6} \mathrm{H}_{5}\right)_{3} \mathrm{H}^{+}$ & \\
2240 & P-H.(Str) $v$ & $(\mathrm{~s})$ \\
851 & P-H .(Str) $v$ & $(\mathrm{~m})$ \\
1471 & P (C6H5).(Str) $v$ & $(\mathrm{~s})$ \\
1579 & C6H6.(Str) $v$ & (m.w) \\
3057 & $=$ C-H.(str) $v$ & $(\mathrm{~s})$ \\
1426 & c=c.(Str) $v$ & $(\mathrm{w})$ \\
497 & $=$ C-H.(Ben) $v$ & $(\mathrm{~s})$ \\
& {$[\text { CrF }]^{-}$} & \\
542 & Cr-F.(Str) & (s) \\
607 & Cr-F.(Str) & (s) \\
687 & Cr-F.(Str) & (s)
\end{tabular}




\subsection{Computational Method}

We applied the DFT method to optimize and calculate molecular properties of synthesized compounds. For DFT, Becke's three-parameter exchange functional was used in combination with the Lee-Yang-Parr correlation functional (B3LYP) with LANL2DZ basis set. Ionic molecule was used without any symmetry restriction. Calculations were done in the gas phase. After the optimization procedures, frequency calculations were done to extract vibrational mode and tested the correctness of true minima. The vibrational frequencies and intensities (spectra) and the eigenvectors for the normal modes were corrected with the appropriate factor and displayed on a computer screen to identify the dominating motions. Moreover, list most important observed vibrational modes of ionic liquids in $400-4000 \mathrm{~cm}^{-1}$ range is presented in Table 2.

Table 2. Comparison of the experimental and calculated vibration data $\left(\mathrm{cm}^{-1}\right)$.

\begin{tabular}{lrr}
\hline Vibration & Experimental & Calculated \\
\hline P-H.(Str) $v$ & 2240 & 2402 \\
P-H.(Str) $v$ & 851 & 882 \\
P(C6H5).(Str) $v$ & 1471 & 1474 \\
C6H6.(Str) $v$ & 1579 & 1627 \\
$=$ C-H(str) $v$ & 3057 & 3250 \\
C=c.(Str) $v$ & 1429 & 1469 \\
=C-H.(Ben) $v$ & 497 & 530 \\
Cr-F.(Str) & 542 & 569 \\
Cr-F.(Str) & 607 & 627 \\
Cr-F.(Str) & 687 & 689 \\
\hline
\end{tabular}

Table 3. The calculated bond lengths $[\AA]$ and bond angles $\left[{ }^{\circ}\right]$ of $\left[\mathrm{CrF}_{4}\right]^{-}$.

\begin{tabular}{cccc}
\hline Bond & Bond lengths $[\AA]$ & Angles & Bond angles [ $\left.{ }^{\circ}\right]$ \\
\hline $\operatorname{Cr}(1)-\mathrm{F}(2)$ & 1.854 & $\mathrm{~F}(2)-\operatorname{Cr}(1)-\mathrm{F}(3)$ & 101.086 \\
$\operatorname{Cr}(1)-\mathrm{F}(3)$ & 1.81 & $\mathrm{~F}(2)-\operatorname{Cr}(1)-\mathrm{F}(4)$ & 104.576 \\
$\operatorname{Cr}(1)-\mathrm{F}(4)$ & 1.81 & $\mathrm{~F}(2)-\operatorname{Cr}(1)-\mathrm{F}(5)$ & 134.49 \\
$\operatorname{Cr}(1)-\mathrm{F}(5)$ & 1.855 & $\mathrm{~F}(3)-\operatorname{Cr}(1)-\mathrm{F}(4)$ & 109.683 \\
$\operatorname{Cr}(1)-\mathrm{F}(2)$ & 1.854 & $\mathrm{~F}(3)-\operatorname{Cr}(1)-\mathrm{F}(5)$ & 104.711 \\
& & $\mathrm{~F}(4)-\operatorname{Cr}(1)-\mathrm{F}(5)$ & 101.129 \\
\hline
\end{tabular}


As shown in Table 2, all reported frequencies corresponding to vibrational mode and the magnitude of these frequencies agree with other similar compounds. In most cases, frequencies in this ionic liquid are nearly the same and the difference between their frequencies is very small. We applied the Gaussian 98 program and the molecule was optimized by the DFT method using B3LYP/LANL2DZ basis set (Table 3).

\section{Results and Discussions}

The salt/Lewis acid adducts usually result in either ionic liquids or crystalline materials with low melting points. Salts containing large organic cations, such as butylpyridinium chloride or 1, 3- dialkyl imidazolium chloride, interact with $\mathrm{CrF}_{3}$ to form ionically conducting liquids at room temperature. Upon melting, $\mathrm{CrF}_{3}$ appears as a molecular liquid with high vapor pressure. It is well known that the melting point of $\mathrm{CrF}_{3}$ can be lowered upon mixing with RF ( $\mathrm{R}$ represents an alkali metal or organic cation), which is believed to originate from the Lewis acid-base interactions of $\mathrm{CrF}_{3}$ with $\mathrm{RF}$ and the formation of large-sized complex. From the binary phase diagram, it is found that a low-lying eutectic occurs in the 2:1 composition of $\mathrm{CrF}_{3}-\mathrm{RF}$. Melting temperature of the eutectic is well below that of the $\mathrm{CrF}_{3}$, representing the minimum liquidus temperature throughout the entire system.

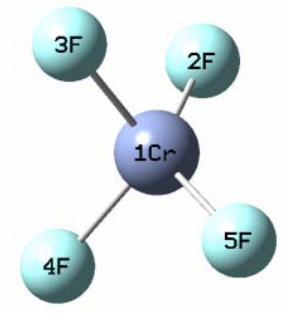

Fig. 2. Optimized structure of $\left[\mathrm{CrF}_{4}\right]^{-}$anion.

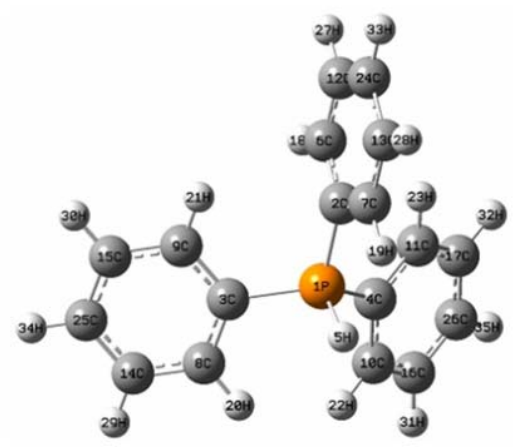

Fig. 3. Optimized structure of $\left[\mathrm{P}\left(\mathrm{C}_{6} \mathrm{H}_{5}\right)_{3} \mathrm{H}\right]^{+}$cation.

We continue to focus on the synthesis and characterization of various ionic liquids. In this paper, we report the synthesis of the new ionic liquid containing (TPPTFC) tetrafluorochromate $\left[\mathrm{CrF}_{4}\right]^{-}$anion and triphenylphosphonium $\left.\left[\mathrm{P}\left(\mathrm{C}_{6} \mathrm{H}_{5}\right)_{3} \mathrm{H}\right]^{+}\right)$cation). Ionic liquid was synthesized through a one-step reaction. Our procedure for producing ionic liquid has some advantages. For example, there is no side product in preparing ionic liquid in our method, the reaction is quite fast and does not require any severe conditions such as high pressure or high temperature, and it is not sensitive to air. The synthesis of the 
TPPTFC has shown that borate was useful for organic chemists. These are analog of the above copper compounds.

$$
\left[\mathrm{P}\left(\mathrm{C}_{6} \mathrm{H}_{5}\right)_{3} \mathrm{H}\right]^{+}\left[\mathrm{CrF}_{4}\right]^{-} \longrightarrow \mathrm{P}\left(\mathrm{C}_{6} \mathrm{H}_{5}\right)_{3}+\mathrm{HF}+\mathrm{CrF}_{3}
$$

The cations and anions are commonly assumed to be in a hypothetical gaseous free state and without any pre-assumed symmetry, but some calculations also involve better approximations to real systems. The structure of the optimized TPPTFC is depicted in (Figs. 2 and 3).

After synthesis of TPPTFC, it was identified in different solvents. This compound was soluble in solvents like DMSO, THF, toluene, acetonitrile, acetone and ether and insoluble in water, ethanol, methanol and hexane. In the FTIR spectra of TPPTFC the strong bands were assigned to the $\mathrm{P}-\mathrm{H}$ and $-\mathrm{C}-\mathrm{H}$ stretching vibrations respectively. The FTIR spectra of TPPTFC showed weak bands corresponding to Cr-F.

After the optimization procedures for obtaining geometry with a minimum energy the vibrational frequencies and intensities and the eigenvectors for the normal modes are calculated and displayed on a computer screen, to identify the dominating motions.

This compound produces big crystal like bulks as shown in Fig. 4, but it is not suitable for single crystal diffraction.

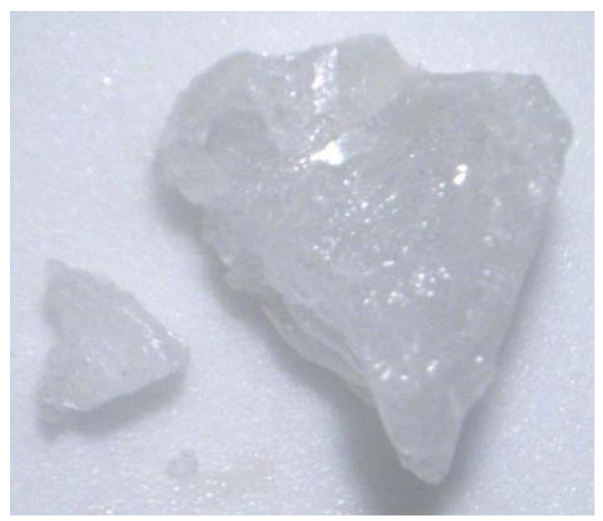

Fig. 4. Crystal graph of $\left[\mathrm{P}\left(\mathrm{C}_{6} \mathrm{H}_{5}\right)_{3} \mathrm{H}\right]^{+}\left[\mathrm{CrF}_{4}\right]^{-}$in acetonitrile solvent.

TGA has been applied to investigate the thermal behavior and structure of TPPTFC. Thermal decomposition of TPPTFC showed multistage processes and results also showed that this IL has excellent thermal stability below $100^{\circ} \mathrm{C}$ (Fig. 5). 


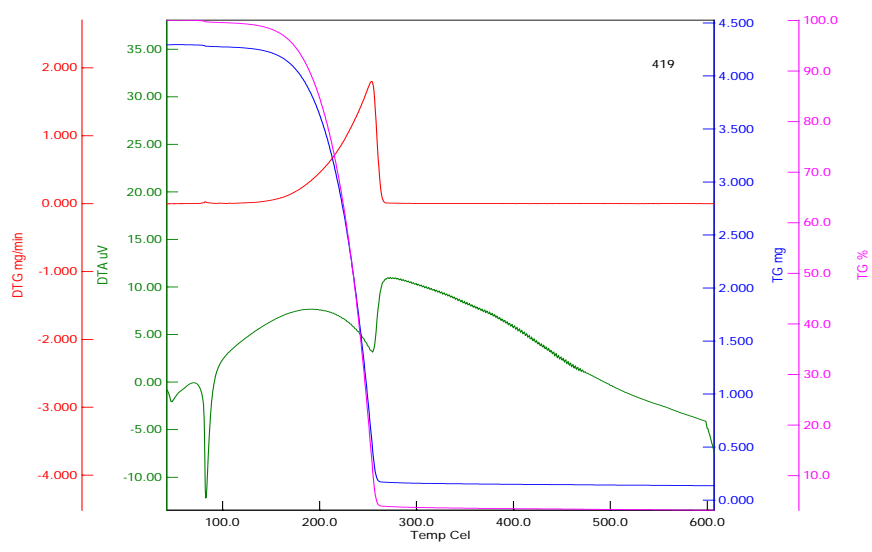

Fig. 5. TGA graph of $\left[\mathrm{P}\left(\mathrm{C}_{6} \mathrm{H}_{5}\right)_{3} \mathrm{H}\right]^{+}\left[\mathrm{CrF}_{4}\right]^{-}$

TPPTFC was further characterized by cyclic voltammetry (CV) and the results showed that this IL compound has reversible behavior and can be used as solvent in many temperate reactions (Fig. 6).

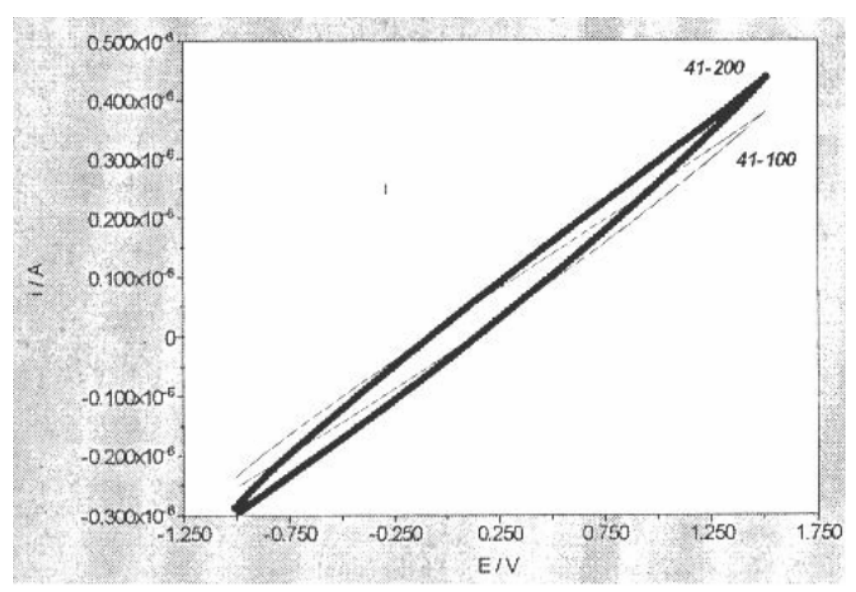

Fig. 6. Cyclic Voltammetry (CV) of $\left[\mathrm{P}\left(\mathrm{C}_{6} \mathrm{H}_{5}\right)_{3} \mathrm{H}\right]^{+}\left[\mathrm{CrF}_{4}\right]^{-}$.

\section{Conclusion}

In this work, a novel chromate ionic liquid with formula $\left[\mathrm{P}\left(\mathrm{C}_{6} \mathrm{H}_{5}\right)_{3} \mathrm{H}\right]^{+}\left[\mathrm{CrF}_{4}\right]^{-}$was synthesized from the reaction of triphenylphosphine with $\mathrm{CrF}_{3}$ and HF. The structure of the compound has been calculated and optimized by the density functional theory (DFT) 
based method at B3LYP/6-311G levels of theory, using the Gaussian 98 package of programs. A comparison between theory and experiment was made. This compound produced big crystal like bulks, but it was not suitable for single crystal diffraction. The $\mathrm{CV}$ indicated that IL has reversible behavior and can be used as solvent in many temperate reactions.

\section{Acknowledgment}

The authors wish to express their warm thanks to Dr. Gh. Rezaei Behbahani for his valuable discussions. This work was supported by Islamic Azad University, Ardabil Branch.

\section{References}

1. B. Linclau, A. K. Sing, and D. P. Curran, J. Org. Chem. 64, 2835 (1999). http://dx.doi.org/10.1021/jo9823442

2. T. Walton, Chem. Rev. 99, 2071 (1999). http://dx.doi.org/10.1021/cr980032t

3. M. J. Earle and K. R. Seddon, Pure. Appl. Chem. 7, 72 (2000).

4. A. R. Hajipour, A. Rajaei, and A.E. Ruoho, Tetrahedron. Lett. 50, 708 (2009). http://dx.doi.org/10.1016/j.tetlet.2008.11.111

5. H. Wang, Y. Liu, Z. Li, X. Zhang, S. Zhang, and Y. Zhang, Eur. Poly. J. 45, 1535 (2009). http://dx.doi.org/10.1016/j.eurpolymj.2009.01.025

6. X. Han and D. W. Armstrong, Acc. Chem. Res. 40, 1079 (2007). http://dx.doi.org/10.1021/ar700044y

7. M. Anouti, M. Caillon-Caravanier, Y. Dridi, J. Jacquemin, C. Hardacre, and D. Lemordant, J. Chem. Thermodyn. 41, 799 (2009). http://dx.doi.org/10.1016/j.jct.2009.01.011

8. P. K. S. Antharjanam, M. Jaseer, K. N. Ragi, and E. Prasad, J. Photochem. Photobiol. A: Chem. 203, 50 (2009). http://dx.doi.org/10.1016/j.jphotochem.2008.12.023

10. G. Garcí-Miaja, J. Troncoso, and L. Romaní, J. Chem. Thermodyn. 41, 161(2009). http://dx.doi.org/10.1016/j.jct.2008.10.002

11. K. A. Kurnia, C. D. Wilfred, and T. J. Murugesan, J. Chem. Thermodyn. 41, 517 (2009). http://dx.doi.org/10.1016/j.jct.2008.11.003 